\title{
Controlling the Diameter of a Pure Electron Plasma to Produce an Exact Two-Fluid Plasma State in a Nested Trap
}

\author{
Toshiki KATO, Haruhiko HIMURA, Shinji SOWA and Akio SANPEI \\ Department of Electronics, Kyoto Institute of Technology, Matsugasaki, Kyoto 606-8585, Japan
}

(Received 20 December 2018 / Accepted 15 January 2019)

\begin{abstract}
Methods for controlling accurately the diameter $d_{e}$ of a pure electron plasma in a nested trap of the BX-U machine are described. By controlling the acceleration voltages and the number of electron guns activated, we can successfully change $d_{e}$ over the range between 0.28 and $2.27 \mathrm{~cm}$. Therefore, $d_{e}$ can be made almost exactly the same as the diameter of a lithium ion plasma enabling the investigation of a two-fluid plasma state by using non-neutral plasmas.
\end{abstract}

(c) 2019 The Japan Society of Plasma Science and Nuclear Fusion Research

Keywords: two-fluid plasma, non-neutral plasma, nested trap

DOI: $10.1585 /$ pfr.14.1201039

A two-fluid plasma $[1,2]$ is an extended magnetohydrodynamic (MHD) model that is widely used for explaining microscopic plasma phenomena that cannot be explained by conventional one-fluid MHD. In a two-fluid plasma, the velocity fields of the ion and electron plasmas are determined by separate fluid equations of motion. Also, the densities of the ions and electrons may differ from each other. However, such a two-fluid plasma stateespecially one in differentially rotating equilibrium [3] has never been verified in laboratory experiments. To investigate the two-fluid plasma state experimentally, we have tested it by using pure lithium-ion $\left(\mathrm{Li}^{+}\right)[4]$ and electron $\left(\mathrm{e}^{-}\right)$plasmas. These are so-called non-neutral plasmas [5]. They can be relaxed into separate rotating thermal equilibria, which rotate rigidly in opposite azimuthal directions, owing to the different signs of their charges. These properties provide definite initial conditions for the $\mathrm{Li}^{+}-$ ion and $\mathrm{e}^{-}$plasmas before superimposing them to produce a two-fluid plasma state.

In our first series of superimposition experiments [6], two problems emerged to be solved. First, the densities of the ion $n_{i}$ and electron $n_{e}$ plasmas could not be controlled, an essential requirement for controlling the ratio of $n_{i}$ to $n_{e}$ precisely. Second, the diameter $d_{e}$ of the $\mathrm{e}^{-}$plasma was much smaller than that $d_{i}$ of the $\mathrm{Li}^{+}$-ion plasmas, which breaks the implicit assumption $d_{e}=d_{i}$ employed in theoretical studies of two-fluid plasmas. In the present paper, we describe methods for solving these two experimental problems.

Experiments to superimpose $\mathrm{Lii}^{+}$-ion plasma on an $\mathrm{e}^{-}$plasma have been conducted in the BX-U linear trap $[7,8]$ as shown in Fig. 1. Five guns are installed on the left-hand side of the tap. A sample of $\beta$-eucryptite is installed on the machine axis to emit $\mathrm{Li}^{+}$-ions, while all four thermionic $\mathrm{e}^{-}$emission cathodes are installed $2.3 \mathrm{~cm}$ away from the machine axis [7]. The number of cathodes used to produce the $\mathrm{e}^{-}$plasma varies. However, in most cases, three cathodes are activated, because the three $\mathrm{e}^{-}$ beams merge quickly and form an $\mathrm{e}^{-}$plasma in the short time of $\approx 200 \mu$ s [7]. The positions of these cathodes can be also varied; however, all are fixed in the presented research. From these guns, $\mathrm{Li}^{+}$-ions and $\mathrm{e}^{-}$beams launched toward the central part of the BX-U machine, where a set of multi-ring electrodes is installed. By applying an independent potential to each electrode, a nested trap $[9,10]$ can be formed there, as shown in Fig. 2. The BX-U trap contains a uniform axial magnetic field up to $0.13 \mathrm{~T}$, which confines both the $\mathrm{Li}^{+}$-ions and the $\mathrm{e}^{-}$plasmas radially. The positive and negative potential wells trap [11] the $\mathrm{Li}^{+}$-ion and the $\mathrm{e}^{-}$plasmas separately but also simultaneously [12], as shown in Fig. 2 (a). We have not yet investigated experimentally the shape of the $\mathrm{e}^{-}$plasma trapped in the negative harmonic potential well of the nested BX-U trap. However, the $\mathrm{e}^{-}$plasma lasts for at least $5 \mathrm{~s}$, which is much longer than the electron-electron binary collision time $(\approx 8 \mathrm{~ms}$ for $T_{e} \approx 15 \mathrm{eV}$ and $n_{e} \approx 5.3 \times 10^{13} \mathrm{~m}^{-3}$ ). We therefore expect the $\mathrm{e}^{-}$plasma to be relaxed into rotational thermal equilibrium in the negative harmonic potential well, which strongly suggests that the shape of the $\mathrm{e}^{-}$plasma is a spheroid. Subsequently, we translated the $\mathrm{Li}^{+}$-ion plasma

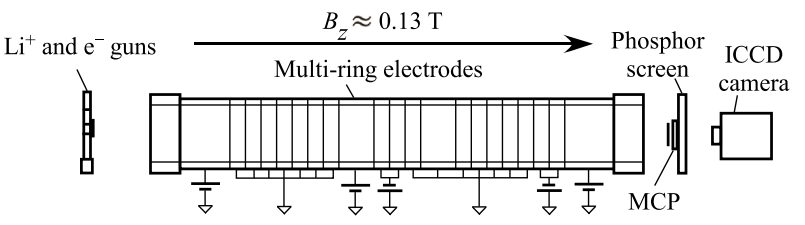

Fig. 1 Schematic of the BX-U linear trap. 


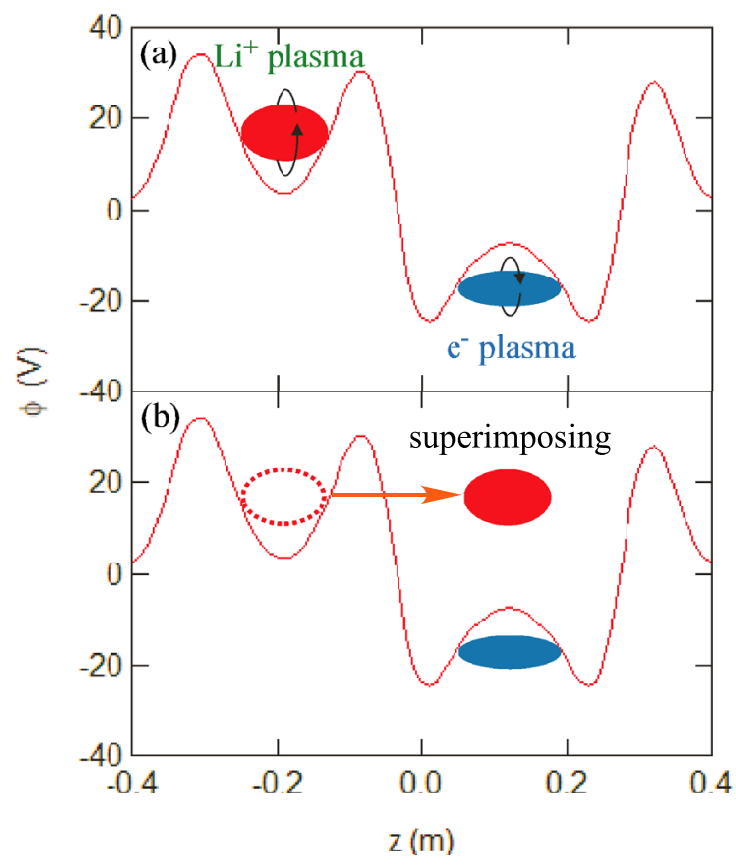

Fig. 2 The plasma-superpositioning sequence in the nested trap. (a) First, $\mathrm{Li}^{+}$-ion and $\mathrm{e}^{-}$plasmas are confined until each relaxes separately into thermal equilibrium in the respective positive and negative potential wells. (b) Second, the $\mathrm{Li}^{+}$-ion plasma is superimposed onto the $\mathrm{e}^{-}$plasma to create a two-fluid plasma state.
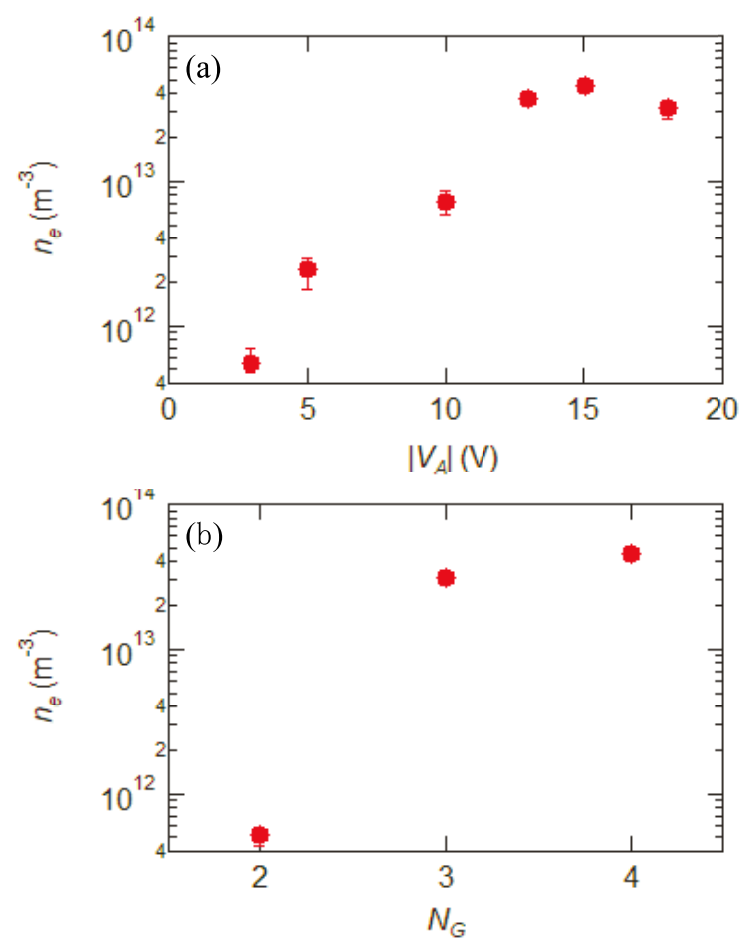

Fig. 3 Dependence of $n_{e}$ on (a) $V_{A}$ and (b) $N_{G}$. The error bars represent the maximum and minimum observed values.

into the nested trap where the $\mathrm{e}^{-}$plasma is confined, as shown in Fig. 2 (b). For the duration of this superimposition, a two-fluid plasma state is thus generated experi-

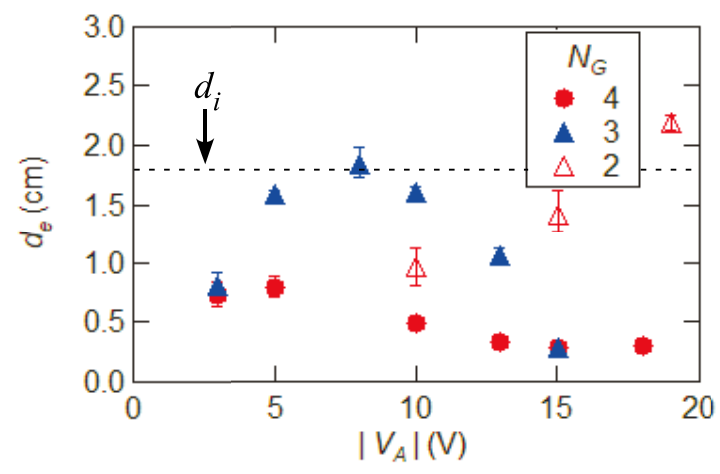

Fig. 4 Dependence of $d_{e}$ on $V_{A}$ and $N_{G}$. For the case where $N_{G}=3$ and $V_{A}=-8 \mathrm{~V}$, we obtain $d_{e}=1.84 \mathrm{~cm}$, which is approximately equal to the diameter $d_{i}=1.77 \mathrm{~cm}$ of $\mathrm{Li}^{+}$-ion plasmas shown by the dashed line. The error bars represent the maximum and minimum observed values.

mentally. For diagnostics, we employ a microchannel plate (MCP) followed by a phosphor screen [13] . Images taken by a high-speed ICCD camera placed outside the vacuum vessel appear on the screen consecutively [14]. Using image analysis [13], we obtained the changes in the twodimensional shapes of both the $\mathrm{Li}^{+}$-ion and $\mathrm{e}^{-}$plasmas after the formation of the two-fluid plasma state. We use the full width at half maximum of the one-dimensional luminosity profile along a horizontal line [4] passing through the center of the $\mathrm{e}^{-}$plasma as the value of $d_{e}$. This is the same procedure as the method to determine the diameter $d_{i}$ of pure $\mathrm{Li}^{+}$-ion plasma confined in the $\mathrm{BX}-\mathrm{U}$ [4].

The values of $d_{i}$ and $d_{e}$ are strongly correlated with the plasma frequency [15]. Since the plasma frequency depends on $n_{i}$ (or $n_{e}$ ) [16], $d_{i}$ (or $d_{e}$ ) can thus be controlled by controlling $n_{i}$ (or $n_{e}$ ). Figure 3 shows the dependence of $n_{e}$ on the accelerating voltage $V_{A}$ and the number $N_{G}$ of $\mathrm{e}^{-}$guns activated. The density $n_{e}$ is clearly changed by varying $V_{A}$ and $N_{G}$. Figure 4 shows the dependence of $d_{e}$ on $V_{A}$ and $N_{G}$, demonstrating that $d_{e}$ can be changed by controlling $V_{A}$ and $N_{G}$. The holding time of $\mathrm{e}^{-}$plasma is variable, but it is fixed at $5 \mathrm{~s}$ in these experiments.

The data on the left-hand side of Fig. 5 show twodimensional integrated distributions for the $\mathrm{e}^{-}$plasmas, while those on the right-hand side show one-dimensional distributions of particle numbers along dashed lines that pass through the corresponding center of the $\mathrm{e}^{-}$plasmas. In fact, $d_{e}$ increases from (a) $0.28 \mathrm{~cm}$ to (b) $1.84 \mathrm{~cm}$ as $n_{e}$ decrease from $\approx 5 \times 10^{13} \mathrm{~m}^{-3}$ to $\approx 7 \times 10^{11} \mathrm{~m}^{-3}$. For the case $N_{G}=3$ and $V_{A}=-8 \mathrm{~V}$, we obtain $d_{e}=1.84 \mathrm{~cm}$. On the other hand, by changing $V_{A}$ applied to the $\mathrm{Li}^{+}$gun, $n_{i}$ can be controlled from $\approx 2 \times 10^{11} \mathrm{~m}^{-3}$ to $\approx 2 \times 10^{12} \mathrm{~m}^{-3}$ without changing $d_{i}(\approx 1.77 \mathrm{~cm})$. In that case, the difference between $d_{i}$ and $d_{e}$ is only $4 \%$. Currently, we are accumulating data on the two-fluid plasma state obtained by using the control methods described above. These data will be reported elsewhere. 
(a)

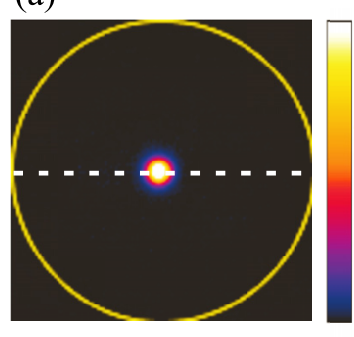

(b)
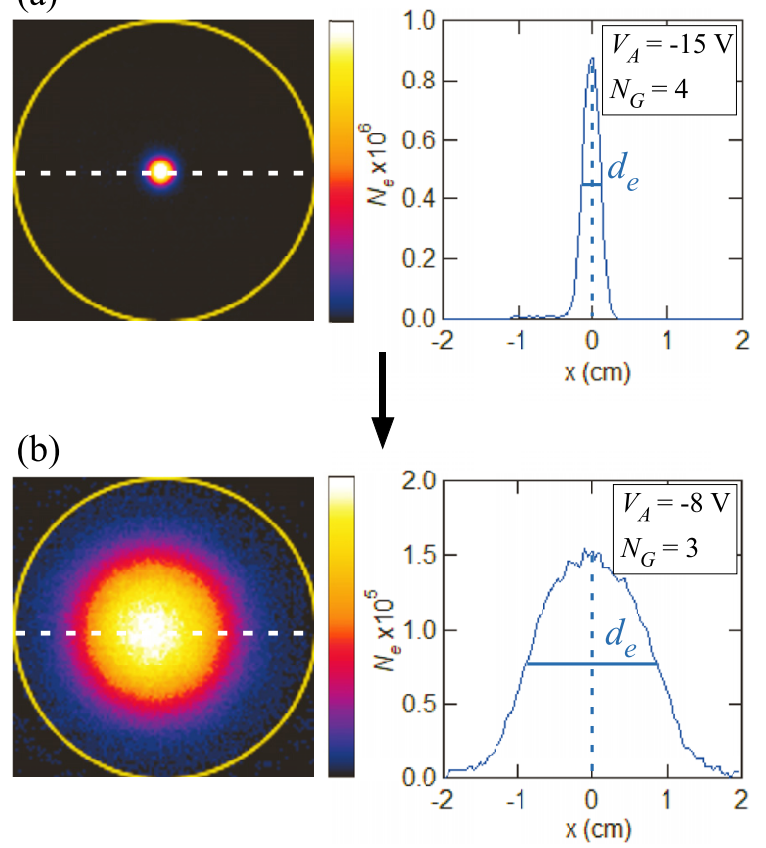

Fig. 5 Images and one-dimensional distributions of the particle number $N_{e}$ for $\mathrm{e}^{-}$plasmas for cases where (a) $V_{A}=-15 \mathrm{~V}$ and $N_{G}=4$ and (b) $V_{A}=-8 \mathrm{~V}$ and $N_{G}=3$.

The authors are grateful to the BX-U group, especially K. Akaike and T. Okada for their help with these experiments. They would like to acknowledge Profs. S. Okada and S. Masamune for their comments. This work is supported by JSPS KAKENHI Grant No. 18H01194.

[1] U. Shumlak, R. Lilly, N. Reddell, E. Sousa and B. Srinivasan, Comput. Phys. 182, 1767 (2011).

[2] H. Himura, AIP Conf. Proc. 1928, 020005 (2018).

[3] R.C. Davidson, Physics of Nonneutral Plasmas (World Scientific Publishing Company, 2001) p. 227.

[4] S. Kawai, H. Himura et al., Phys. Plasmas 23, 022113 (2016).

[5] D. Walsh and D.H.E. Dubin, Phys. Plasmas 25.5, 052119 (2018).

[6] S. Yamada, H. Himura, T. Kato, S. Okada, A. Sanpei and S. Masamune, AIP Conf. Proc. 1928, 020016 (2018).

[7] H. Himura, Nucl. Instrum. Methods Phys. Res., Sect. A 811, 100 (2016).

[8] H. Himura, S. Yamada, T. Kato and S. Masamune, Plasma Fusion Res. 12, 1201037 (2017).

[9] C. Amsler, G. Bonomi, A. Fontana, A. Kellerbauer, V. Lagomarsino, E. Rizzini, A. Rotondi, G. Testera, L. Venturelli and N. Zurlo, Int. J. Mod. Phys. A 29, 1430035 (2014).

[10] C. Ordonez, D. Dolliver, Y. Chang and J. Correa, Phys. Plasmas 9, 3289 (2002).

[11] G. Gabrielse et al., Phys. Lett. A 129, 1 (1998).

[12] H. Himura et al., Plasma Fusion Res. 8, 2401017 (2013).

[13] H. Himura, S. Nakata and A. Sanpei, Rev. Sci. Instrum. 87, 063306 (2016).

[14] S. Yamada and H. Himura, Rev. Sci. Instrum. 87, 036109 (2016).

[15] M. Chaker and M. Moisan, J. Appl. Phys. 57.1, 91 (1985).

[16] Y. Okamoto, J. Vac. Soc. Jpn. 59, 161 (2016). 